\title{
Discectomia percutânea a laser
}

\author{
José Jorge Facure*
}

Setor de Pós-graduação da Área de Neurologia da Faculdade de Ciências Médicas da Unicamp

\section{RESUMO}

Analisamos os resultados da discetomia percutânea a laser em 27 pacientes com hérnia discal lombar. O tratamento é feito com anestesia local, em âmbito ambulatorial. Para a punção do disco, que é feita sob controle radiológico, utiliza-se uma agulha especial com mandril cuja retirada permite a introdução de uma fibra óptica de $800 \mu$ de diâmetro, que conduzirá o laser. Para o procedimento, utilizamos o equipamento Diodo Laser Sharplan 6020. Foi aplicado, em média, um total de 1.800 joules de energia distribuído em três pontos do núcleo pulposo. Para cada ponto realizamos uma série de cinco seqüências de disparos-laser, cada série correspondendo a oito pulsos-laser de 15 watts, com duração de um segundo, atingindo-se 600 joules de energia. O período de seguimento foi de 1 a 38 meses, média de 8 meses. Os resultados foram considerados bons, com desaparecimento da dor e retorno do paciente ao mesmo trabalho anterior em $66,7 \%$ dos casos.

Concluímos que a discectomia percutânea a laser é um método efetivo que deve ser considerado como alternativa no tratamento da hérnia discal lombar.

\section{PALAVRAS-CHAVE}

Discectomia percutânea a laser. Hérnia de disco. Laser.

\section{ABSTRACT}

Percutaneous laser lumbar disc decompression

This study is an analysis of the outcome of percutaneous laser disc decompression in 27 patients with lumbar disc herniation. We used a Diodo Laser System, Sharplan 6020. On the average it was applied a total energy of about 1800 joules in single shots (1 second, 15 watts). These shots were applied in 3 points of the nucleus pulpous in a series of 5 pulses trains, each containing 8 pulses lasers, 1 second on, 1 second off (600 joules). The patients were followed-up for 1 to 38 months, the average follow-up being 8 months.

Successful outcome, which means absence of pain and return to previous work, was achieved in $66,7 \%$ of the cases.

It is concluded that percutaneous laser decompression is effective and must be considerated as an alternative method in the treatment for lumbar disc herniation.

\section{KEYWORDS}

Laser diskectomy. Lumbar disc herniation. Laser.

\section{Introdução}

O tratamento da hérnia discal lombar por meio da evaporação parcial do núcleo pulposo com o uso de irradiação laser foi realizada pela primeira vez em $1986^{4}$. Desde então, diversos autores publicaram suas experiências demonstrando que a redução da pressão dentro do disco intervertebral pela evaporação a laser, de uma porção do núcleo pulposo, seria suficiente para produzir o desaparecimento da sintomatologia em cerca de $64,6 \%$ a 78,4\% dos $\operatorname{casos}^{1,3,5,6}$. Ascher ${ }^{1}$, pioneiro na realização desse método, já com casuística de 509 casos, aponta $75 \%$ de bons resultados. Entretanto, essa nova tecnologia tem limitações, não sendo indicada nos casos de hérnia expulsa, estreitamento do canal radicular, espondilolistese, processo degenerativo discal avançado com presença de ar no disco ou cirurgia convencional recente no mesmo nível. Além do que, a punção do espaço L5/S1 não é conseguida em todos os casos. Esses autores salientam que o resultado está condicionado a uma indicação cirúrgica inteligente e que o procedimento deva ser feito somente após um período de aproximadamente três meses de tratamento clínico. $\mathrm{O}$ fato de ser um procedimento ambulatorial

* Prof. Adjunto Livre-docente de Neurocirurgia. 
denominado minimamente invasivo, realizado com anestesia local e realmente efetivo, o torna, sem dúvida, útil no manuseio da hérnia discal lombar. É objetivo desse trabalho analisar os benefícios da discectomia a laser como recurso terapêutico no tratamento da hérnia discal lombar.

\section{Casuística e método}

Nossa casuística compreende 27 pacientes com hérnia discal lombar, sem fenômenos degenerativos (osteoartrose) significativos, submetidos a microdiscectomia percutânea a laser. Os pacientes foram operados em regime ambulatorial, no Hospital Centro Médico de Campinas, no período de 11 de junho de 1996 a 1 de agosto de 1999. O diagnóstico foi confirmado por tomografia computadorizada (TC) em 20 pacientes; por ressonância magnética (RM) em seis e um doente já nos foi encaminhado com mielografia confirmando hérnia discal em L2/L3. A idade variou de 21 a 60 anos (média de 46 anos e mediana de 43 anos). Dezesseis pacientes eram do sexo masculino e 11 do sexo feminino. Em três pacientes a hérnia localizava-se em nível L5/S1; em um caso, a hérnia se localizava no espaço L2/L3 e, nos demais, no espaço L4/L5.

\section{Técnica cirúrgica}

O paciente é posicionado em decúbito lateral, com o lado da hérnia para cima. $O$ ponto de punção demarcado aproximadamente entre $8 \mathrm{~cm}$ a $10 \mathrm{~cm}$ acima da linha média e na altura da crista ilíaca para o espaço L4/L5. O local exato da punção, bem como o ângulo de penetração da agulha, é dado por controle radiológico (intensificador de imagem). Após a anestesia no local previamente demarcado e asséptico, introduzimos agulha ( $\left.\mathrm{n}^{\circ} 18\right)$ com angulação de $50^{\circ}$ a $60^{\circ}$, procurando penetrar no disco em posição rente à faceta articular e em ponto eqüidistante dos planos ósseos. Em primeiro lugar, a agulha é colocada a $1 \mathrm{~cm}$ para frente do centro do disco. Retira-se o mandril e introduz-se uma fibra óptica de $800 \mu$, previamente demarcada para se posicionar a $5 \mathrm{~mm}$ além da extremidade da agulha. Nesse ponto, utilizando-se de um equipamento diodo laser (Sharplan 6020) realizamos oito disparos de 15 watts com duração de um segundo e com intervalos de um segundo. Nesse mesmo ponto, repetimos o procedimento por cinco vezes, o que atinge a energia de 600 joules. Com controle radiológico, recuamos a agulha para o centro do disco e repetimos o mesmo procedimento. Como última etapa, recuamos a agulha para
$1 \mathrm{~cm}$ aquém do centro do disco e novamente repetimos o procedimento. Isto equivale ao uso total no núcleo pulposo de 1.800 joules, em média.

Após o procedimento, o paciente permanece no ambulatório por 2 a 3 horas e em seguida é encaminhado para o domicílio.

\section{Resultados}

O período de seguimento foi de 1 mês a 38 meses, média de 8 meses. Para 18 pacientes, o resultado foi considerado bom (66,7\%): completo alívio da dor ciática e da lombalgia, volta ao mesmo trabalho anterior, ocasionalmente lombalgia de pequena intensidade não limitante. Em cinco pacientes (18,5\%), o resultado foi considerado regular: melhora da dor ciática, crises de lombalgia freqüentes, às vezes limitantes e ocasionalmente lombociatalgia aos esforços. Para quatro pacientes $(14,8 \%)$, o resultado foi considerado mau: sem melhora da sintomatologia.

Dentre estes pacientes nos quais o resultado do tratamento foi considerado mau, um submeteu-se a cirurgia convencional na cidade de origem após dois meses; outro paciente continuou a queixar-se da mesma sintomatologia, porém a RM realizada após um mês da discectomia percutânea a laser não demonstrou hérnia discal; os outros dois com hérnia discal em L4/L5, havia persistência da dor logo após o procedimento a laser. Entretanto, em um, tomografia computadorizada demonstrou hérnia em L5/S1 e, no espaço L4/L5, onde foi realizada a discectomia a laser não havia hérnia. Este paciente não retornou para seguimento; o último caso evoluiu com períodos de melhora e piora, tendo sido diagnosticado um ano após a discectomia a laser, recidiva de hérnia L4/L5 e estreitamento do canal radicular. Esse paciente foi encaminhado para cirurgia convencional.

Em sete pacientes foi possível realizar estudo pósoperatório: com TC em cinco e com RM em dois. Em um paciente com boa evolução clínica, realizamos tomografia computadorizada 1 mês e 18 dias após a discectomia percutânea a laser, tendo sido evidenciado tênue contorno discal, sem a imagem marcante da hérnia (Figuras 1 e 2).

\section{Discussão}

A técnica para a descompressão percutânea do disco intervertebral lombar foi publicada em 1992, em 


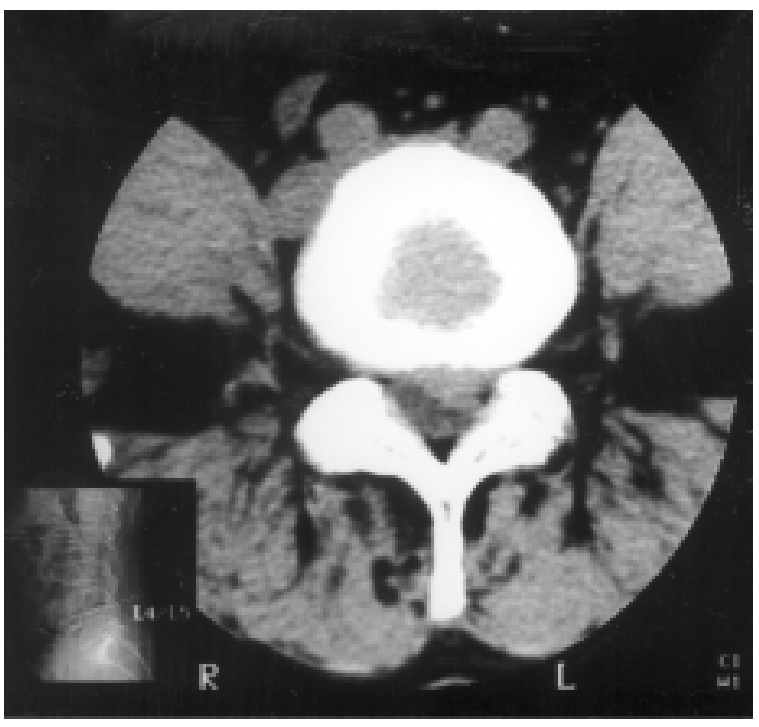

Figura 1 - TC mostrando hérnia discal em L4/L5.

um trabalho conjunto, desenvolvido por Peter W. Ascher, na Universidade de Graz, Áustria e por Daniel S. Choy e cols., na Universidade de Colúmbia, New York $^{5}$. O procedimento é feito com anestesia local (lidocaína a 2\%). Para o acesso aos discos L2/L3, L3/ L4 e L4/L5, o paciente é colocado em decúbito lateral com o lado da dor radicular para cima. $\mathrm{O}$ ponto da punção se situa $8 \mathrm{~cm}$ a $10 \mathrm{~cm}$ acima da linha média, na altura da crista ilíaca para o nível L4/L5. O ponto exato a ser anestesiado para a penetração da agulha é localizado por radioscopia. Utiliza-se uma agulha específica $\mathrm{n}^{\circ} 18$, de $20 \mathrm{~cm}$ de comprimento que, além das aberturas nas extremidades, possui uma abertura lateral para a saída do vapor, produto da irradiação a laser. O ângulo de penetração da punção é de $50^{\circ}$ a $60^{\circ}$ em relação à linha média. Todo o procedimento para se atingir o centro do disco é feito sob controle fluoroscópico nos planos lateral e ântero-posterior. A agulha é dirigida para o centro do disco e, com a angulação descrita, geralmente atinge-se, em primeiro lugar, a faceta articular, após o que, é dirigida alguns milímetros lateralmente, procurando-se entrar no disco com a agulha rente à faceta. $\mathrm{O}$ ângulo de penetração não pode ser muito pequeno, senão facilmente a raiz será atingida. O disco deve ser penetrado com a agulha entre a raiz lateralmente e a faceta articular medialmente. Essa técnica possibilita facilmente a punção de L2/L3, L3/L4 e L4/L5. A punção do disco no espaço L5/S1 pela via póstero-lateral é mais difícil, principalmente nos casos em que esse nível está muito abaixo da crista ilíaca, pois o osso ilíaco dificulta que a agulha atinja o espaço. Para a punção do espaço L5/S1, os autores utilizam uma agulha curva para acompanhar a curvatura doosso

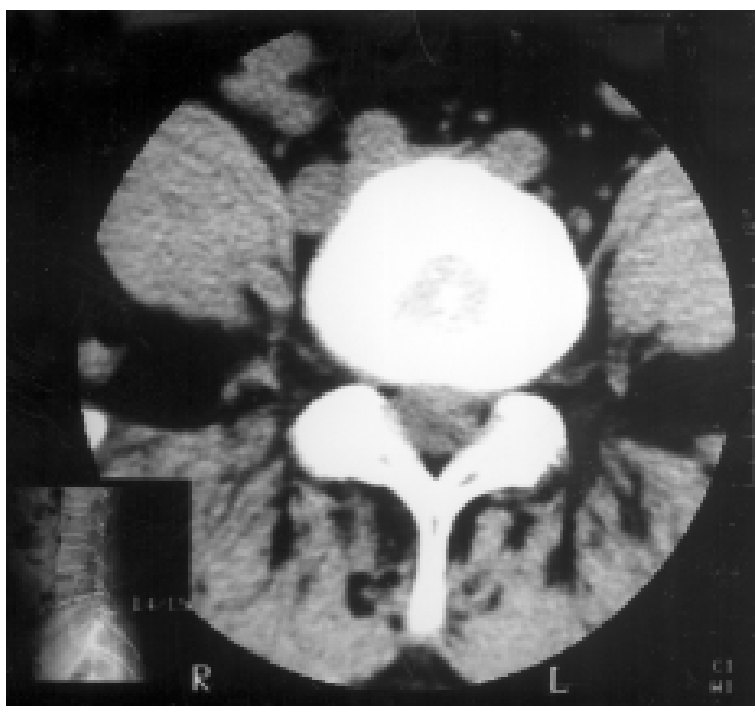

Figura 2 - TC realizada 1 mês e 18 dias após a discectomia percutânea a laser. Nota-se o desaparecimento da herniação e tênue contorno do disco.

ilíaco. Ascher ${ }^{1}$ refere que, em cerca de $20 \%$ dos pacientes, não conseguiu realizar a punção em L5/S1. Nesses casos, os pacientes são encaminhados para cirurgia convencional. A partir de 1994, Choy ${ }^{4}$ passou a utilizar a via posterior extradural para atingir o espaço L5/S1. A punção é feita a $2 \mathrm{~cm}$ da linha média e o ângulo exato para que a agulha atinja o centro do disco é orientado por radioscopia.

Para os nossos pacientes não temos indicado a punção posterior para o espaço L5/S1 como procedimento de rotina. Quando a posição desse nível não permite a punção póstero-lateral com pequena curvatura na agulha, temos preferido encaminhar o paciente para a microdiscectomia convencional. Essa conduta visa não submeter o paciente ao risco de punção intratecal com a agulha específica para o laser, que é de grosso calibre.

Após o posicionamento da agulha, o mandril é removido e uma fibra óptica é introduzida em uma extensão previamente demarcada, ultrapassando em 5 mm a extremidade da agulha.

Na primeira publicação, os autores localizavam a agulha a $1 \mathrm{~cm}$ posterior ao centro do disco, concentrando, neste ponto, a energia de 1.000 a 1.850 joules. Atualmente, o total da energia é distribuída em três pontos: $1 \mathrm{~cm}$ posterior ao centro do disco, no centro do disco e $1 \mathrm{~cm}$ anterior ao centro do disco, seguindose esta ordem para os disparos do laser.

$\mathrm{O}$ fundamento científico para o uso do raio laser baseia-se nos experimentos de Nachemson ${ }^{11,12}$, que demonstrou que pequenos volumes de líquido injetados dentro do disco intervertebral provocam 
grandes aumentos da pressão intradiscal. O autor comparou o disco intervertebral como sendo um espaço hidráulico fechado, com o núcleo pulposo que é um gel mucoprotéico, contido pelo anel fibroso e por dois planos cartilaginosos rígidos. Experimentalmente, demonstrou que discos de cadáveres humanos apresentam uma pressão de $1.175 \mathrm{mmHg}$. Demonstrou também que o disco intervertebral não é facilmente deformado pelo aumento da pressão, significando que a ocorrência de aumento de pressão intradiscal não se dissipa facilmente. Realizando a introdução de $1 \mathrm{ml}$ de solução salina em 17 discos intactos de cadáveres humanos, constatou um aumento médio de $2.340 \mathrm{mmHg}$ na pressão intradiscal (Gráfico 1).

Baseado nesta observação, $\mathrm{Choy}^{5}$ propôs a evaporação de pequeno volume do núcleo pulposo como possibilidade terapêutica para a hérnia de disco lombar. Em experimentação com disco bovino, Choy e cols. ${ }^{5}$ demonstraram que a irradiação do núcleo pulposo com

\section{Pressão intradiscal mmHg}

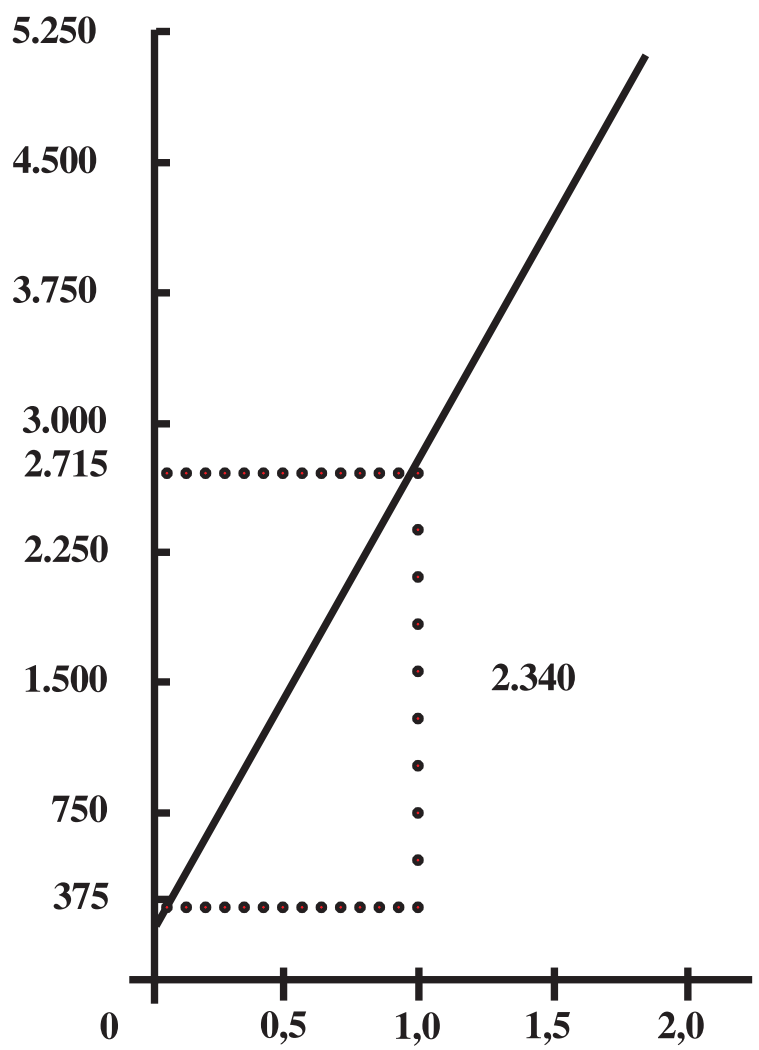

Solução salina - ML

Gráfico 1 - Variação da pressão intradiscal em função do volume de solução salina injetado no interior do disco. laser Nd:YAG (neodyinium:ytrium-alminium-garnet), com 40 watts, com pulsos de $0,4 \mathrm{seg}$, em seqüência de 40 pulsos, correspondendo a uma liberação de energia de 640 joules, produz uma lesão de $10 \mathrm{~mm}$ x $2 \mathrm{~mm}$ de diâmetro, o que corresponde a uma evaporação de 31,46 $\mathrm{mm}^{3}$, que correspondem a 32,22 mg. Em experiência subseqüente, demonstraram que com o uso do laser $\mathrm{Nd}$ :YAG com 20 watts de potência aplicados através de fibra óptica com extremidade hemisférica, utilizandose pulsos-laser com duração de 1 segundo, com pausa de 1 segundo, em um total de 50 pulsos, liberando, portanto, uma energia de 1.000 joules, produzia lesão intradiscal elíptica de $10 \mathrm{~mm} \times 4 \mathrm{~mm}$ de diâmetro, evaporando-se $63 \mathrm{~mm}^{3}$.

Para demonstrar a queda da pressão intradiscal com a evaporação a laser, Choy e cols. ${ }^{5}$ elaboraram um modelo experimental, medindo a pressão intradiscal em 18 discos intactos de cadáveres. Com o modelo experimental os discos eram submetidos a uma pressão de sobrecarga, partindo-se de uma pressão intradiscal inicial de $2.419 \mathrm{mmHg}$. Foi realizada a irradiação de 1.000 joules, com duração de 10 minutos, com laser Nd: YAG, através de uma fibra óptica colocada no centro do disco. Do total dos 18 discos utilizados para o estudo, no primeiro minuto da aplicação do laser houve um ligeiro aumento da pressão intradiscal em sete. Essa ocorrência foi interpretada como tendo havido um bloqueio da extremidade da agulha pela carbonização, dificultando a saída de vapor. Para os 11 casos restantes, a pressão manteve-se estável no primeiro minuto. Nos próximos 9 minutos da aplicação do laser, a pressão intradiscal caiu para $1.346 \mathrm{mmHg}(44,3 \%) \mathrm{e}$ continuou a cair até 23 minutos após o término da aplicação do laser. A pressão se estabilizou em 1.075 $\mathrm{mmHg}$, demonstrando-se uma queda significativa de $55,6 \%$.

Choy e Diwan ${ }^{6}$, também em 1992, realizaram a medida da pressão intradiscal em quatro pacientes com hérnia discal submetidos ao tratamento com o laser $\mathrm{Nd}: \mathrm{YAG}$, utilizando-se 1.000 joules de energia. Para o primeiro paciente, a pressão inicial intradiscal foi de $300 \mathrm{mmHg}$ e, após a aplicação do laser, caiu para $154 \mathrm{mmHg}$, ou $43,8 \%$. Nos três pacientes subseqüentes, utilizaram método hidráulico, considerado mais sensível para medir a pressão intradiscal. Nesse método, obtém-se a relação da pressão inicial com a pressão final e a relação encontrada nos três casos foi de $7: 3 ; 5: 3$; e $4: 2$, confirmando uma queda em torno de $50 \%$ da pressão inicial em todos, e confirmando a eficácia do laser para provocar a queda da pressão intradiscal in vivo.

Choy e cols. ${ }^{5}$ publicaram, em 1992, os resultados do tratamento da hérnia discal lombar com o uso da discectomia a laser em 333 pacientes, tendo sido tratados 377 discos (226 em L4/L5; $119 \mathrm{em} \mathrm{L5/S1;30} \mathrm{em}$ $\mathrm{L} 3 / \mathrm{L} 4 \mathrm{e} 2 \mathrm{em} \mathrm{L} 2 / \mathrm{L} 3)$. O período maior de seguimento foi 
de 62 meses sendo, a média, de 26 meses. Os pacientes foram triados para a cirurgia, com o uso de um protocolo de admissão em que se destacam os seguintes pontos principais: comprovação dignóstica por tomografia computadorizada ou ressonância magnética de hérnia discal não-expulsa; correspondência clínica com a neuroimagem; sem melhora com o tratamento clínico no mínimo por três meses; sem espondiloartrose, sobretudo sem estreitamento do canal radicular; sem cirurgia anterior para hérnia de disco; sem intercorrências clínicas como angina, doença hemorrágica, infarto do miocárdio recente ou insuficiência cardíaca; pacientes sem litígios pendentes e, finalmente, referem que os pacientes e as testemunhas assinam um termo de consentimento informado. Foi utilizado o laser Nd:YAG com 20 a 23 watts, com pulso-laser de 1 segundo, seguido de uma pausa de 1 segundo, com um total de 1.000 a 1.850 joules de energia. Esse último valor foi utilizado para pacientes com altura maior que $180 \mathrm{~cm}$ e peso maior que $85 \mathrm{~kg}$. Se o paciente apresentasse dor ou sensação de calor no início da aplicação do laser, as pausas entre os disparos do laser eram prolongadas para 3 a 6 segundos. A fibra óptica era removida da agulha para facilitar a saída do vapor. A fase de aplicação do laser durou, em média, 3 a 4 minutos. O paciente saía da mesa cirúrgica deambulando, com ajuda, e recebia orientação para limitar suas atividades por três dias. Para 261 pacientes $(78,4 \%)$ os resultados foram considerados bons ou regulares. Em 166 desses pacientes, o alívio da dor foi constatada durante a aplicação do laser. Em 72 doentes $(21,6 \%)$, os resultados foram desfavoráveis. Todos esses 72 pacientes foram encaminhados para cirurgia convencional. $\mathrm{O}$ tratamento com o laser não exclui, portanto, a possibilidade de o paciente necessitar de discectomia convencional. Houve a necessidade de repetição do laser em 11 pacientes, com benefícios para sete $(64 \%)$. Os autores admitem que um dos fatores para os casos com maus resultados foi a existência de severa degeneração discal, com "fenômeno de vácuo" no núcleo pulposo. Esse fato torna o disco um sistema hidráulico menos homogêneo, reduzindo os efeitos benéficos da redução da pressão intradiscal. Acreditam também que alguns pacientes operados com TC ou RM, realizados em vários locais com qualidade variável de imagem, não deveriam ter sido incluídos no estudo. Admitem que em seis pacientes com maus resultados o laser não foi utilizado com energia suficiente ( 400 a 600 joules). Em apenas $1 / 3$ dos casos, aproximadamente, o estudo pósoperatório com RM, realizado 3 a 4 meses após o tratamento com laser, demonstrou modificação anatômica do disco considerada como modesta a moderada. Nota-se, portanto, uma dissociação entre a imagem da RM e a melhora do quadro clínico.
Recentemente, em 1997, Tomami e cols. ${ }^{13}$ realizaram estudo com ressonância magnética 24 horas após o tratamento da hérnia discal com laser Nd:YAG. Verificaram que a modificação de sinal dentro do disco aumenta muito após o tratamento, mas apenas em sete pacientes verificaram modificações consideradas como lesões produzidas pelo laser (trato laser). Não encontraram nenhuma correlação dessas pequenas alterações observadas na RM com a evolução clínica.

Ascher ${ }^{1}$, em 1995, apresentou os resultados obtidos em 509 pacientes com hérnia discal lombar, tratados com o uso do laser Nd:YAG. Na maioria das vezes, o paciente revela alívio da dor ciática ao serem atingidos os primeiros 300 joules. O total de energia utilizada variou entre 1.152 a 1.826 joules. O procedimento é feito ambulatorialmente e, uma semana após a aplicação, ainda há um alívio progressivo da dor. Admite que isso se deve à progressão das modificações estruturais no núcleo pulposo na área adjacente à lesão provocada pelo laser. Refere também que o procedimento a laser é indicado para os pacientes que não responderam ao tratamento clínico e que isto corresponderia a cerca de $10 \%$ a $20 \%$ dos casos. Do total dos 509 pacientes, houve melhora em $75 \%$, tendo sido registrado um caso de discite, como uma única complicação. Os restantes $25 \%$ foram encaminhados para cirurgia convencional e, em apenas $50 \%$ desses, a cirurgia demonstrou patologia convincente para a persistência da dor: hérnia subligamentar, fragmento de disco livre, canal lombar ou radicular estreitos e espondilolistese.

A partir de julho de 1995, Ascher ${ }^{2}$ passou a trabalhar com novo tipo de laser que utiliza um semicondutor, o diodo-laser (SHARPLAN 6020). Foi demonstrado também por diversos autores ${ }^{8,9,10,14}$ que esse tipo de laser tem a mesma eficácia do Nd:YAG e é gerado por um aparelho compacto, totalmente portátil e com custo muito menor. Apresentou uma casuística com 26 casos de hérnia de disco, tendo sido utilizado 15 watts por segundo, com um total em torno de 2.000 joules. Utilizando-se uma fibra óptica hemisférica de $800 \mu$, essa energia é distribuída em três pontos do disco. Para cada ponto realiza uma seqüência de 40 a 48 disparos-laser de 15 watts por segundo com pausa de 1 segundo (600 a 720 joules). Nesse grupo de 26 casos, não ocorreu complicação. Em alguns casos houve queixas de dor nas costas que foi atribuída à sensação de calor no anel fibroso ou no pericôndrio; em outros casos, houve referência a aumento inicial da dor ciática que foi atribuída ao aumento transitório da pressão intradiscal determinado pela evaporação da água. Porém, de modo geral, o que se observa é um desaparecimento da dor ciática logo após a aplicação de cerca de 300 joules e o estiramento da perna não provoca mais a dor Aproximadamente 20 minutos após 
o procedimento, os pacientes receberam alta hospitalar. Não conseguiu realizar o procedimento em sete pacientes: não foi possível puncionar o espaço L5/S1 em três; em dois casos houve reação alérgica ao anestésico e, em outros dois, o procedimento foi interrompido devido à dor excessiva.

Wyman e cols. ${ }^{14}$, em 1992, publicaram estudo comparativo entre a eficácia do Nd:YAG e do diodo laser. Estudaram a ação dos dois tipos de lasers em tecidos humanos, úteros e cólons de pacientes submetidos à histerectomia e à colectomia por diversas patologias. A aplicação do laser era realizada in vitro através de fibra óptica em contato com a mucosa. Demonstraram que a lesão provocada pelos dois tipos de lasers são equivalentes.

Choy e Ngeon 7 , em 1998, relataram a utilização da discectomia a laser para tratamento de estenose do canal lombar, em casos em que a protrusão discal é um elemento significativo nesse estreitamento. Em uma série de 35 pacientes, referiram resultados excelentes em $69 \%$, tendo sido avaliada apenas a manifestação dolorosa.

\section{Conclusões}

Nossa casuística, embora pequena, demonstra que a discectomia percutânea a laser, por ser um procedimento minimamente invasivo e efetivo, visto que determina melhora imediata da sintomatologia, abreviando o tempo de enfermidade em cerca de $66,7 \%$ dos casos, deve ser considerada como opção no tratamento da hérnia discal lombar.

\section{Referências}

1. ASCHER PW: Percutaneous lumbar discectomy. In: Schimidek HH, William HS (eds): Operative Neurosurgical Techniques. Ed 3. Philadelphia, WB Saunders Company, 1995, vol 2, pp 1927-34.
2. ASCHER PW: The Diodo laser as a tool for nucleus pulposus denaturation (NPN). Apresentado no Curso Discectomia Percutânea Lombar Assistida por Laser, organizado pela Universidade Federal de São Paulo, Escola Paulista de Medicina e pelo Centro de Estudos do Hospital Israelita Albert Einstein, São Paulo, 10 e 11 de maio de 1996.

3. BOTSFORD JA: Radiological considerations: patient selection for percutaneous laser disc decompression. J Clin Laser Med Surg 12:255-9, 1994.

4. CHOY DSJ: The problem of the L5-S1 disc solved by needle entry with an extrathecal approach. J Clin Laser Med Surg 12:321-4, 1994.

5. CHOY DSJ, ASCHER PW, SADDEKNI S, ALKAITIS D, LIEBLER W, HUGHES J, DIWAN S, ALTMAN P: Percutaneous laser disc decompression. Spine 17:949-56, 1992.

6. CHOY DSJ, DIWAN S: In vitro and in vivo fall of intradiscal pressure with laser disc decompression. J Clin Laser Med Surg 10:435-7, 1992.

7. CHOY DSJ, NGEOW J: Percutaneous laser disc decompression in spinal stenosis. J Clin Laser Med Surg 16:123-5, 1998.

8. JUDY MM, MATHEWS JL, ARONOFF BL, HULTS DF: Soft tissue studies with $805 \mathrm{~nm}$ diodo laser radiation: thermal effects with contact tips and comparison with effects of $1064 \mathrm{~nm} \mathrm{Nd:YAG} \mathrm{laser} \mathrm{radiation.} \mathrm{Lasers}$ Surg Med 13:528-36, 1993.

9. JACQUES SL, RASTAGAR S, MOTAMEDI M, THOMPSON S, SCHWARTZ J, TORRES J, MANNOMEN I: Liver photocoagulation with diode laser ( 805 $\mathrm{nm})$ vs. Nd:YAG laser (1064 nm). SPIE:Laser-Tissue Interaction III (Los Angeles) 1646:107-17, 1992.

10. MANNI J: Surgical diodo lasers. J Clin Laser Med Surg 10:377-80, 1992.

11. NACHEMSON A: The influence of spinal movements on the lumbar intradiscal pressure and on the tensile stresses in the annulus fibrosus. Acta Orthop Scand 33:183-207, 1961.

12. NACHEMSON A: The effects of forward leaning on lumbar intradiscal pressure. Acta Orthop Scand 35:314-28, 1965.

13. TONAMI H, YOKOTA H, NAKAGAWA T, HIGASHI K, OKIMURA T, YAMAMOTO I, NISHIJIMA Y: Percutaneous laser discectomy: MR findings within the first 24 hours after treatment and their relationship to clinical outcome. Clin Radiol 52:938-44, 1997.

Original recebido em outubro de 1999

Aceito para publicação em dezembro de 1999

\section{Endereço para correspondência:}

José Jorge Facure

Rua Onze de Agosto, 412

CEP 13013-100 - Campinas, SP 